\title{
Estructura y composición florística del bosque ribereño subandino de la subcuenca de Yumbillo, Yumbo (Valle del Cauca)
}

\section{Structure and floristic composition of the sub-Andean coastal subbasin of Yumbillo, Yumbo (Valle del Cauca)}

\author{
${ }^{1}$ Carlos Gustavo Chaves Campo y ${ }^{2}$ Shirley Andrea Rodríguez Espinosa \\ ${ }^{1}$ Ingeniero Agroforestal. ${ }^{2}$ Ingeniera Forestal \\ E-mail: 1'carlos.chavez@unad.edu.co, ${ }^{2}$ shirley.rodriguez@unad.edu.co \\ 1, ${ }^{2}$ Escuela de Ciencias Agrícolas, Pecuarias y del Medio Ambiente. \\ Universidad Nacional Abierta y a Distancia (UNAD). Palmira. Valle del Cauca (Colombia)
}

\begin{abstract}
Resumen
Se analizaron la estructura y la composición florística del bosque ripario subandino en la subcuenca del río Yumbillo, Municipio de Yumbo (Valle del Cauca), vertiente oriental de la Cordillera Occidental de los Andes, Colombia. Mediante 20 parcelas de $100 \mathrm{~m}^{2}$, en el rango altitudinal 1500-2235 msnm se registraron datos de individuos con diámetros a la altura del pecho $(\mathrm{DAP}) \geq 10 \mathrm{~cm}$, composición florística y estructura vertical y horizontal, mediante cálculo de abundancia, frecuencia, dominancia e índice de valor de importancia (IVI). La composición florística se constituyó de 825 individuos distribuidos en 93 especies, 71 géneros y 50 familias botánicas; las familias dominantes fueron Myrtaceae, Lauraceae, Melastomataceae, Moraceae, Euphorbiaceae, Fagaceae, Acanthaceae y Tiliaceae. La especie de mayor peso ecológico fue el Truco (Hyeronima $s p$ ) y las de mayor frecuencia fueron el Truco (Hyeronima scabrida), el Roble (Quercus humboldtii) y el Higuerón de nacimiento (Ficus apollinaris). Los mayores valores de importancia correspondieron a las especies Truco (Hyeronima scabrida), Arrayán (Myrcia sp), Nacedero (Trichanthera gigantea), Otobo (Dialyanthera lehemannii) y Naranjuelo (Lacistema aggregatum). Se categorizó la estructura vertical en 3 estratos, 508 individuos en el I (3 y $16 \mathrm{~m}), 270$ en el II (17 y $23 \mathrm{~m})$ y 47 en el emergente (alturas superiores a $24 \mathrm{~m}$ ). Se concluye que el bosque ripario posee un alto grado de especies pioneras y típicas de bosques en recuperación, donde se destaca el Medio comino (Ocotea $s p$ ), especie maderable amenazada que puede hacer parte de las acciones de restauración ecológica y enriquecimiento florístico en áreas de bosques riparios y en la protección de cuencas.
\end{abstract}

Palabras clave: Bosque, cuenca, restauración ecológica, frecuencia, abundancia, dominancia.

\begin{abstract}
The structure and floristic composition of riparian forest in the sub-Andean basin of the river Yumbillo in the municipality of Yumbo, Valle del Cauca, eastern slope of the western cordillera of the Andes, Colombia were analyzed. By 20 plots of $100 \mathrm{~m} 2$, in the altitude range between 1500 to $2235 \mathrm{~m}$., Data was recorded in individuals with a diameter of a breast height $(\mathrm{DBH}) \geq 10 \mathrm{~cm}$, floristic composition and vertical and horizontal structure by calculation of abundance, frequency, dominance and importance value index (IVI). The floristic composition consisted of 825 individuals in 93 species, 71 genera and 50 botanical families, the dominant families were Myrtaceae, Lauraceae, Melastomataceae, Moraceae, Euphorbiaceae, Fagaceae, and Tiliaceae acanthaceae. The species were heavier ecological Truco (Hyeronima $s p$ ) and the most frequent were Truco (Hyeronima scabrida), Roble (Quercus humboldtii) and the "Higueron de nacimiento" (Ficus Apollinaris). The highest values corresponded to species of importance such as Truco (Hyeronima scabrida) Arrayan (Myrcia sp), Nacedero (Trichanthera), Otobo (Dialyanthera lehemannii) and Naranjuelo (Lacistema aggregatum). Vertical structure was categorized into three strata, 508 individuals in the I (3 and $16 \mathrm{~m}), 270$ in the II (17 and $23 \mathrm{~m})$ and 47 in the Emerging (heights above $24 \mathrm{~m}$ ). It was concluded that the riparian forest has high and typical pioneer species of forest recovery, highlighted the presence of Medio comino (Ocotea sp) threatened timber species that can be part of ecological restoration actions and floristic enrichment in areas of riparian forest and watershed protection.
\end{abstract}

Key words: Forest, watershed, ecological restoration, frequency, abundance, dominance. 


\section{Introducción}

Las subcuencas Yumbillo y Santa Inés conforman la

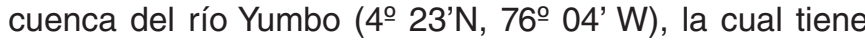
una extensión de 5049,8 ha y una altura máxima de 2250 msnm, y descarga en el río Cauca a los $980 \mathrm{msnm}$. La zona amortiguadora de la cuenca se compone de áreas en recuperación, plantaciones comerciales de coníferas, zonas destinadas a la ganadería y cultivos (medicinales, musáceas y frutales - Lulo, mora, granadilla y tomate de árbol-). La presión antrópica ha generando cambios en el uso de los terrenos, pues ha vulnerado hábitats y nichos de especies de fauna y ocasionado la desaparición de algunas especies florísticas de valor económico y ecológico.

Las diversas fisonomías dibujadas por el mosaico de usos (agrícola, pecuario y forestal), los grados de erosión (que varían desde moderados hasta severos) y la aplicación de técnicas inapropiadas de manejo del suelo presentan el panorama desolador de estas laderas de la Cordillera Occidental (Escobar, 2001). Entre las acciones conducentes a contener la degradación de las coberturas arbóreas naturales en la cuenca, la Corporación Autónoma Regional del Cauca (CVC) declaró dos zonas forestales protectoras en el municipio de Yumbo: una de ellas comprende áreas de los corregimientos de San Marcos, Montañitas, Santa Inés, Mulaló y Yumbillo; la segunda abarca áreas de los corregimientos de Arroyohondo, La Olga y El Pedregal (POT, 2001).

La escasa información sobre la estructura de la vegetación en ambientes riparios de la cuenca del río Yumbo hace necesarios estudios florísticos confiables que permitan formular recomendaciones para el manejo y la conservación. Para contribuir al conocimiento del bosque ripario subandino de la subcuenca del río Yumbillo y a las acciones de ordenamiento de los recursos naturales, esta investigación se propuso analizar la estructura y la composición florística riparias.

\section{Métodos}

\section{Área de estudio}

La subcuenca Yumbillo se localiza en la vertiente oriental de la Cordillera Occidental en el bosque húmedo subandinoandino (1500-2200 msnm), con una temperatura media de $20^{\circ} \mathrm{C}$, precipitación media anual de $1600 \mathrm{~mm}$, régimen bimodal (con marzo-mayo y octubre-noviembre como los periodos más lluviosos) y vegetación de selva andina higrofítica de clima frío (Minambiente, 2006).

La vegetación se caracteriza por la diversidad y la gran cantidad de especies arbóreas, con hojas cartáceas, coriáceas y persistentes, aspectos propios de la selva neotropical. Se identifican bosques secundarios altamente intervenidos, ubicados en los nacimientos de agua o superando los $1500 \mathrm{msnm}$; las mayoría de las especies comerciales han sido extraídas y la extensión de estos relictos selváticos disminuye debido a la presión causada por la ampliación de la frontera agrícola y pecuaria (Londoño, 1997).

El estudio se realizó entre noviembre de 2010 y febrero de 2011. Se trabajó en 20 parcelas de $100 \mathrm{~m}^{2}$ cada una, distribuidas al azar, distanciadas $100 \mathrm{~m}$, agrupadas de acuerdo con el rango altitudinal (Tabla 1) y levantando censos florísticos con registros de especies por estratos. Para cada especie se determinaron su abundancia, su frecuencia, su dominancia y su valor de importancia (IVI) sobre la magnitud y el rol de participación en la comunidad vegetal, lo cual permitió evaluar el peso ecológico de cada especie (Lamprecht, 1990).

Tabla 1. Distribución de parcelas en transectos según rango altitudinal

\begin{tabular}{crr}
\hline Transectos & Altitud $(\mathrm{msnm})$ & Coordenadas planas $(\mathrm{N}-\mathrm{E})$ \\
\hline \multirow{2}{*}{1} & & $1057664-888345$ \\
& & $1058337-888805$ \\
2 & & $1057405-888016$ \\
& & $1057536-888376$ \\
3 & & \\
& & $1056914-887381$ \\
4 & & $1057221-888009$ \\
& $2076-2235$ & $1056687-888152$ \\
& & $1056860-888227$ \\
\hline
\end{tabular}




\section{Resultados y discusión}

\section{Composición florística}

Para la muestra de $2000 \mathrm{~m}^{2}$ la riqueza florística del bosque ripario incluye 93 especies y 71 géneros, distribuidos en 825 individuos.

Se encontraron 50 familias de especies arbóreas conocidas y clasificadas total o parcialmente; las de mayor abundancia fueron: Myrtaceae, Lauraceae,
Moraceae, Euphorbiaceae, Tiliaceae, Melastomataceae, Acanthaceae y Fagaceae, que representan el $50 \%$ del total de familias.

\section{Estructura vertical}

Los transectos mostraron individuos (con DAP $\geq 10 \mathrm{~cm}$ ) en 3 estratos. : I (entre 3 y $16 \mathrm{~m}$ ), II (entre 17 y $23 \mathrm{~m}$ ) y emergente (alturas superiores a $24 \mathrm{~m}$ ) (Tabla 2).

Tabla 2. Número de individuos por estratos evaluados en fragmentos de bosque ripario, en la subcuenca del río Yumbillo, Cuenca del río Yumbo (Valle del Cauca)

\begin{tabular}{ccccc}
\hline \multirow{2}{*}{ Estratos } & \multicolumn{4}{c}{$\begin{array}{c}\text { Transectos } \\
\text { (No. de individuos) }\end{array}$} \\
\cline { 2 - 5 } & $\mathbf{1}$ & $\mathbf{2}$ & $\mathbf{3}$ & $\mathbf{4}$ \\
\hline I & 132 & 116 & 158 & 102 \\
II & 103 & 93 & 27 & 47 \\
Emergente & 4 & 7 & 16 & 20 \\
\hline Total & $\mathbf{2 3 9}$ & $\mathbf{2 1 6}$ & $\mathbf{2 0 1}$ & $\mathbf{1 6 9}$ \\
\hline
\end{tabular}

\section{Estructura horizontal}

\section{Índices de importancia ecológica}

Alvis (2009) indica que los análisis de abundancia, frecuencia y dominancia permiten tener una idea sobre un determinado aspecto estructural del bosque. A pesar del gran valor científico práctico de tales enfoques, ellos suministran informaciones parciales y, hasta cierto punto, aisladas. Por tal razón, se ha sugerido la combinación de estos valores para obtener lo que se ha denominado índice de valor de importancia (IVI), el cual es la suma de abundancia relativa, dominancia relativa y frecuencia relativa (Matteucci y Colma, 1982).

En general, las especies que mostraron ser más abundantes fueron: Arrayán (Myrcia sp), con 62 individuos; Balso blanco (Heliocarpus $s p$ ), con 38; Nacedero (Trichanthera gigantea), con 35; Higuerón de nacimiento (Ficus apollinaris), con 35, y Truco (Hyeronima scabrida), con 30 .

Las especies Truco (Hyeronima scabrida), Roble (Quercus humboldtii) e Higuerón de nacimiento (Ficus apollinaris) estuvieron presentes en todas las parcelas de los transectos; las especies que solo aparecen en una parcela representan el $34,13 \%$; las que están en 2 , el $12,06 \%$; las que están en 3 , el $5,86 \%$, y las que están en 4 parcelas, el 53,08\%. Todo ello indica que hay heterogeneidad en el ecosistema, y no se presenta dominancia de una especie en particular.
Los análisis para encontrar la dominancia o el grado de cobertura de las especies (espacio ocupado por ellas) determinaron que los mayores valores los obtuvieron las especies Cedro rosado (Cedrela angustifolia), Medio comino (Ocotea sp), Eucalipto (Eucalyptus grandis), Vainillo (Senna spectabilis) y Pomarrosa (Syzygium jambos).

\section{Índice de valor de importancia (IVI)}

EI IVI para el transecto 1 destaca el Nacedero (Trichanthera gigantea), con el 43,83\%, y el Arrayán (Myrcia sp), con el $43,53 \%$. Las 2 especies suman el $29,12 \%$ del total del IVI, y se encuentran ampliamente distribuidas en este tipo de bosque, en el rango altitudinal 1500-1640 msnm.

EI IVI para el transecto 2 está representado por el Otobo (Dialyanthera lehemannii), con el 54,91\%, y el Arrayán (Myrcia sp), con el 53,77\%. Las 2 especies suman el $36,22 \%$ del total del IVI, y se encuentran ampliamente distribuidas en este tipo de bosque, a la altura 1670-1875 msnm.

El IVI para el transecto 3 está representado por el Naranjuelo (Lacistema aggregatum), con el $62,90 \%$, y el Cedro cebollo (Cedrela montana), con el 48,89\%. Las 2 especies suman el $37,26 \%$ del total del IVI, y se encuentran ampliamente distribuidas entre los 1927-2005 msnm.

EI IVI para el transecto 4 está representado por el Truco (Hieronyma sp), con el 85,65\%, y el Arrayán (Myrcia sp), 
con el $45,61 \%$. Las 2 especies suman el $43,75 \%$ del total del IVI, y se encuentran ampliamente distribuidas en el bosque de rango altitudinal 2076-2235 $\mathrm{msnm}$.

\section{Cociente de mezcla (CM)}

El índice $\mathrm{CM}$ expresa la relación entre el número de especies y el número de individuos totales (número de individuos/número de especies), y proporciona una idea de la intensidad de mezcla, así como una primera aproximación a la heterogeneidad de los bosques. EI CM del ecosistema evaluado fue de 1:8, lo cual indica que por cada ocho individuos muestreados es posible encontrar una especie diferente. Este tipo de bosque puede, entonces, considerarse heterogéneo.

\section{Conclusiones}

La investigación en 4 transectos de bosque subandino de la subcuenca de Yumbillo permitió conocer que la composición florística está representada en 91 especies, correspondientes a 50 familias y 71 géneros, donde las familias dominantes son Myrtaceae, Lauraceae, Melastomataceae, Moraceae, Euphorbiaceae, Fagaceae, Acanthaceae y Tiliaceae.

Las especies Nacedero (Trichanthera gigantea), Mayo (Tibouchina lepidota), Arrayán (Myrcia sp), Otobo (Dialyanthera lehemannii) y Naranjuelo (Lacistema aggregatum) representan el mayor número de individuos desde el análisis en transecto: (35, 16, 19, 19 y 17, respectivamente), con una abundancia relativa del $14,64 \%$, el $9,47 \%$, el 8,80\%, el 8,80\% y el 8,46\%, respectivamente.

Las especies más abundantes fueron: el Arrayán (Myrcia $s p)$, con 62 individuos; el Balso blanco (Heliocarpus sp), con 38; el Nacedero (Trichanthera gigantea), con 35; el Higuerón de nacimiento (Ficus apollinaris), con 35, y el Truco (Hyeronima scabrida), con 30.

Se encontraron bajos porcentajes de dominancia; sin embargo, sobresalieron las especies Cedro rosado (Cedrela angustifolia) y Medio comino (Ocotea $s p$ ), en relación con el tamaño que alcanzan.

Los resultados de IVI permiten plantear que la estructura del bosque presenta características de ecosistema ripario, en la cual se reflejan el grado de riqueza específica y la presencia de especies típicas y de bosques en estado sucesional.

La vegetación de la subcuenca presenta especies heterogéneas, que conforman un ecosistema frágil, debido a las características del medio (pendiente del terreno, posición fisiográfica, acciones antrópicas, entre otras) y al estado de conservación.

Es relevante la presencia de dos individuos de la especie Medio comino (Ocotea sp.), en vía de extinción, y de la especie Oreja de mula (Ocotea calophylla), que han sido objeto de talas durante muchos años por parte de los aserradores.
Es necesario incentivar la investigación en este tipo de ecosistemas riparios, en las relaciones flora-fauna y en la biología reproductiva de las especies, con el fin de mejorar la comprensión sobre el funcionamiento de los relictos boscosos y agenciar iniciativas de conservación.

Es importante mencionar que en la subcuenca del río Yumbillo se encontró alta presencia de individuos de la especies Higuerón de nacimiento (Ficus apollinaris, Moraceae), Mayo (Tibouchina lepidota, Melastomataceae) y Nacedero (Trichanthera gigantea, Acanthaceae), las cuales juegan un papel ecológico, en la protección, moderan el clima, mejoran la calidad del aire, brindan albergue a la vida silvestre y conservan el agua y así ayudan a que los caudales hídricos beneficien a las comunidades, que usufructúan estos recursos naturales.

\section{Agradecimientos}

Los autores agradecen a Bredio Mosquera, Bernardo Mambuscay y Bertha Mambuscay, habitantes de la cuenca de Yumbo, por su colaboración en el trabajo de campo. Al Ingeniero Forestal Eugenio Escobar y al curador del Herbario Valle Carlos Gutiérrez, de la Universidad Nacional de Colombia (sede Palmira), por el apoyo en la identificación y la clasificación de las muestras botánicas.

\section{Literatura citada}

1. Alvis, J. (2009). Analisis estructural de un bosque natural localizado en la zona rural del municipio de Popayan. Facultad de ciencias agropecuarias, Universidad del Cauca.

2. Escobar, E. (2001). Presentación de Yotoco "Reserva natural'. Palmira: Universidad nacional de Colombia.

3. Lamprecht, H. (1990). Silvicultura en los trópicos.: Hamburgo:Gesellhaft.

4. Londoño, L. (1997). Yumbo nuestra comunidad. Cali: Imprenta departamental del Valle del Cauca.

5. Matteucci, S. \& Colma, A. (1982). Metodología para el estudio de la vegetación. Washington:Secretaría General de la Organización de los Estados Americanos.

6. Ministerio del Medio Ambiente. (2006). Tatamá. Disponible en: http://web.minambiente.gov.co/biogeo/ menu/biodiversidad/regiones/andes/parques/parq_ tatama.

7. POT. (2001). Alcaldía de Yumbo, Valle. Yumbo: Decreto 21 noviembre 2001.

Recibido: 22 de febrero de 2012.

Aceptado: 20 de abril de 2012. 